\title{
Web design and production process analysis
}

\author{
Qiguang Ke \\ Wenzhou Vocational \& Technical College, Wenzhou. 325035 \\ qiguang@163.com
}

Keywords: Web page, Design, Process.

\begin{abstract}
With the continuous development of information age, the number of Internet users in China rises sharply, which has reached about half the population at present. Therefore, various websites also arises at the historic moment, including high-quality goods and dregs. The number of broadband and mobile Internet users is growing, but at the same time, the Internet infrastructure resources of our country (the amount of bandwidth, web pages, domain name, etc.) growth is relatively slow, which requires on web design fully consider the influence of various factors on the network access. Learning the excellent part of website design daringly and exploring the improvement of backward parts actively are of vital importance.
\end{abstract}

\section{Design Overview}

Web design is to express a positive atmosphere in the form of a website, promoting the spirit of exploring and striving for ideals and goals. The hope is to foil this uncompromising breath through the color, layout design and the structure of the web, etc. Design mainly hopes to show the positive spirit with strong colors ,so the main audiences should be young groups whose life is full of passion and dream.

\section{Issues to be solved}

\subsection{From the perspective of color}

Browsing hundred of sites by searching the Internet, the style of color and the design characteristics are analyzed and summarized one by one. The result is they have so problems such as "raw”, "pink" and "dazzling”.

1) The reason of "raw" in color design mainly because the purity of the color is too high, almost all the pure color are used. Therefore, the combination and collocation of color piece is improper, which seems the color piece is incongruent in the whole page. This kind of use only considers the characteristics of the color contrast and conflict, while ignoring the harmony of the color.

2) The reason of "pink" in color design is mainly because there is no obvious color contrast in the interface, which makes people feel unfocused. Because there is no distinction with other colors, it does not achieve the effect of contrast, thus it makes the color of the picture dull and tedious.

3) The appearance of "dazzling" in color design is mainly because the color relation of the interface is not clear: the local color is overemphasized while the overall color relation is ignored. Excessive embellishment not only does not help the website, but also produces a dazzling sensation, which makes the viewer tired of visual fatigue and unable to concentrate on the resources required.

\subsection{Form the perspective of man-machine interaction}

\section{1) Response time}

Many studies have shown that the satisfaction of website users is closely related to the sense of control of the system, and the user's sense of control depends greatly on the response speed of the system. 
2) Link

As the space is too large and the links between the nodes are complicated, it is possible for users to browse the Internet and get lost. The first kind is mainly related to cognitive load.

3) Web title

When designing a web page title, you should pay attention to both the user's attention and the need for search engine retrieval. Take the homepage design of the website as an example. In general, the title of the homepage is the name of the website or the company name.

4) Interface

It is generally believed that interface consistency is mainly reflected in three aspects: the effect of metaphor, the relationship between the axis of the system and the output, the appearance or visual effect of the interface. Some studies have shown that enhanced interface consistency can improve user's performance and satisfaction, while reducing operational errors. However, it is also reported that interface consistency sometimes contradicts the usability and learnability of the interface.

5) Structure type of web page

Through a lot of analysis and comparison, some websites adopt a single flat structure, which is bad for visitors to view relevant websites. Most search engines want to see a flat, tree-like structure. Finally, the advantages of using a tree structure for a web site are as follows: the URL is standardized, the structure is clear, the keyword layout is accurate, and the user experience is consistent.

\section{Website module design}

According to the function of the website, the website can be divided into some modules in the theme of youth, such as article management, video management and message management.

Article management module is the core part of the site. A computer can articles according to the administrator to add, modify, and delete operations, to automatically update (produce) site article information (including images). It is the core module of the website.

Video management module is an auxiliary part of the site. A computer can add, modify, and delete operations according to the video of the administrator, such as the site for automatic updates (produce) video information. It's an add-on to the site.

Message management mode includes the addition, maintenance and query of message information. Message maintenance includes the reply, modification and deletion of the article information (including pictures).

\subsection{Function block}

According to the function of website implementation, the website is divided into article management, video management, and message management, as shown in figure 1.

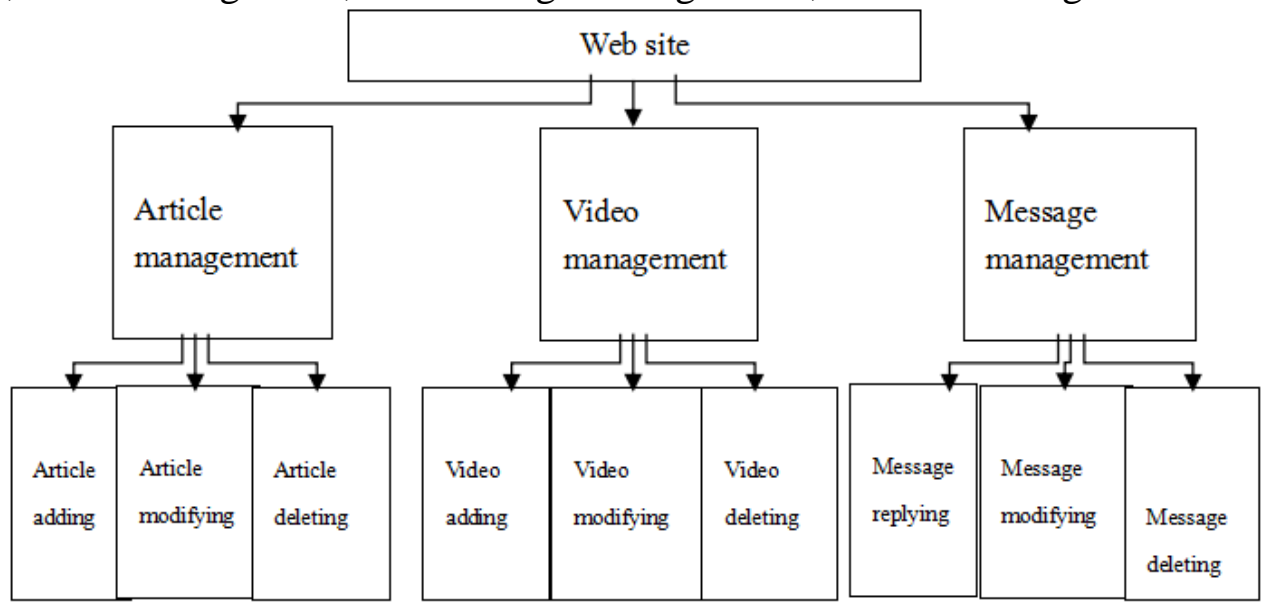

Figure 1: Web function block 


\subsection{Page flow}

Through the above analysis, this website will set up three modules: articles, video, and message. These three roles restrict and interact with each other. The full interaction flow chart is shown in figure 2.

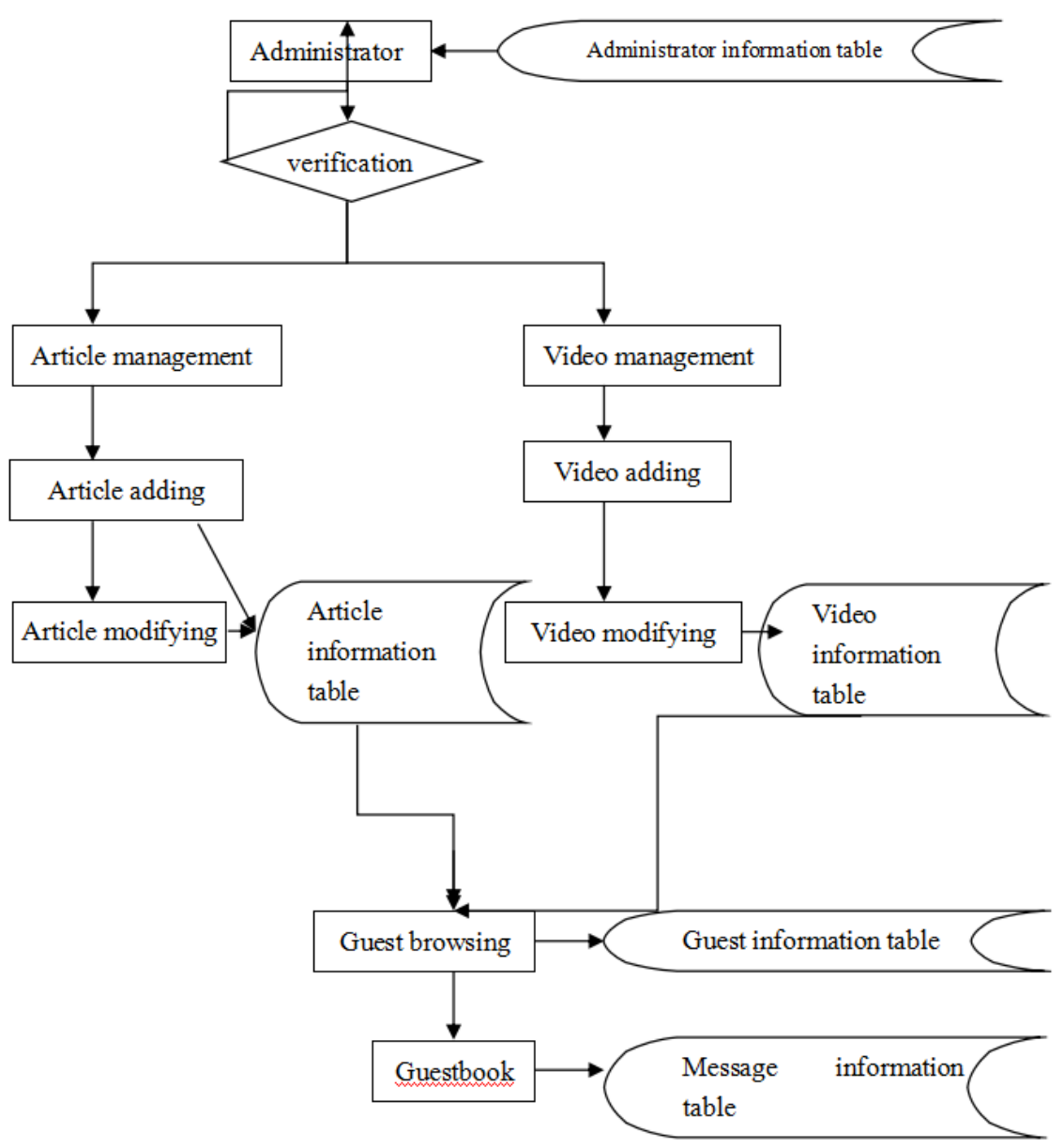

Figure 2: Page flow

\section{WEB PAGE DESIGN}

\subsection{Screen resolution and font design}

1) The display resolution is the number of pixels displayed on the screen. The resolution $160 \mathrm{x}$ 128 means that the horizontal pixels are 160 and the vertical pixels are 128 . The higher the resolution is, the greater the number of pixels are, and the more sophisticated the image is. In the same screen size, the higher the resolution is, the finer and more subtle the effect is .

Currently, 99\% of visitors in China use $1024^{*} 768$ or larger monitors, so this time the page design resolution on youth is $1024 * 768$. Current screen resolution statistics are shown in figure 3 . 


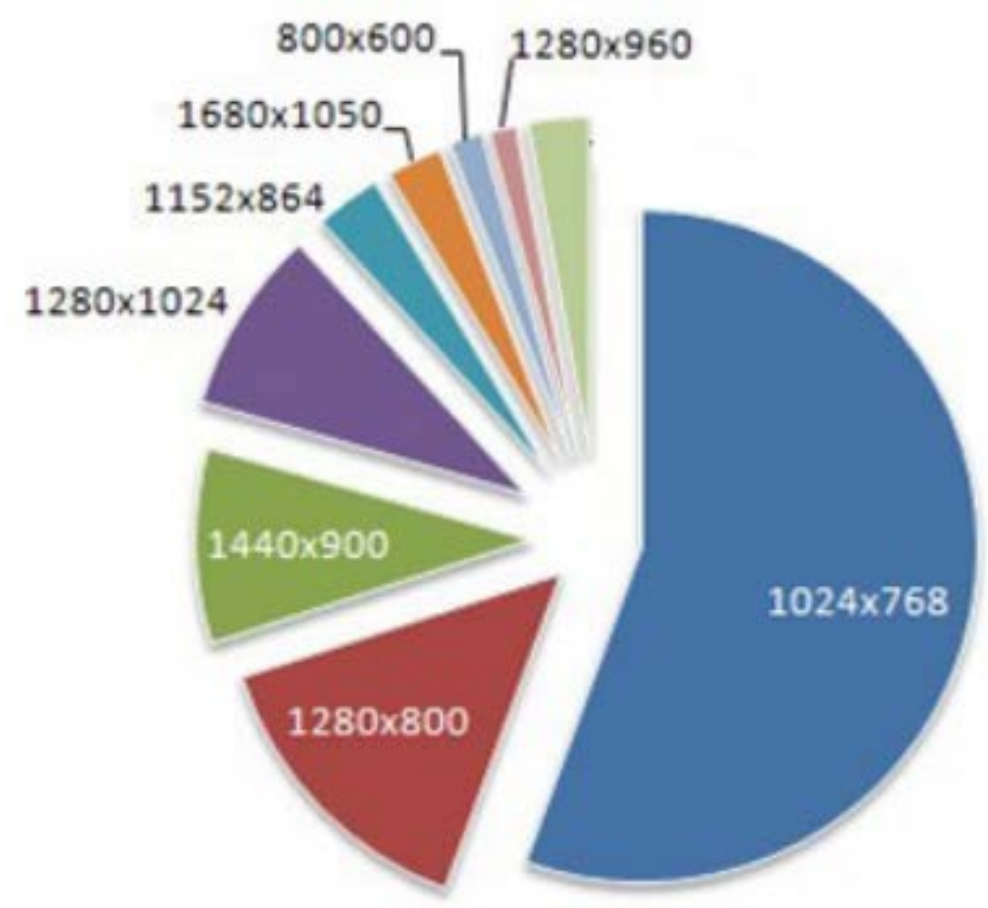

Figure 3: Screen resolution statistics

2) The type and size of the font in the web page are related to a number of factors, such as screen size, resolution, use of people, and so on. Text size is an important part of user experience. Different resolutions, different display sizes, different DPI, and even different browser Settings will have an impact on the final text size. Although many browsers can now easily zoom in and out of the page, the user has to resize every time they visit, which is a problem, and a lot of users don't know how to scale the page. It is better to give users a more considerate default font size based on the user's resolution. What is the appropriate size? Here's a survey: first, the main resolution and screen size of the user, as shown in table 1.

Table 1: Statistical table of resolution and screen size

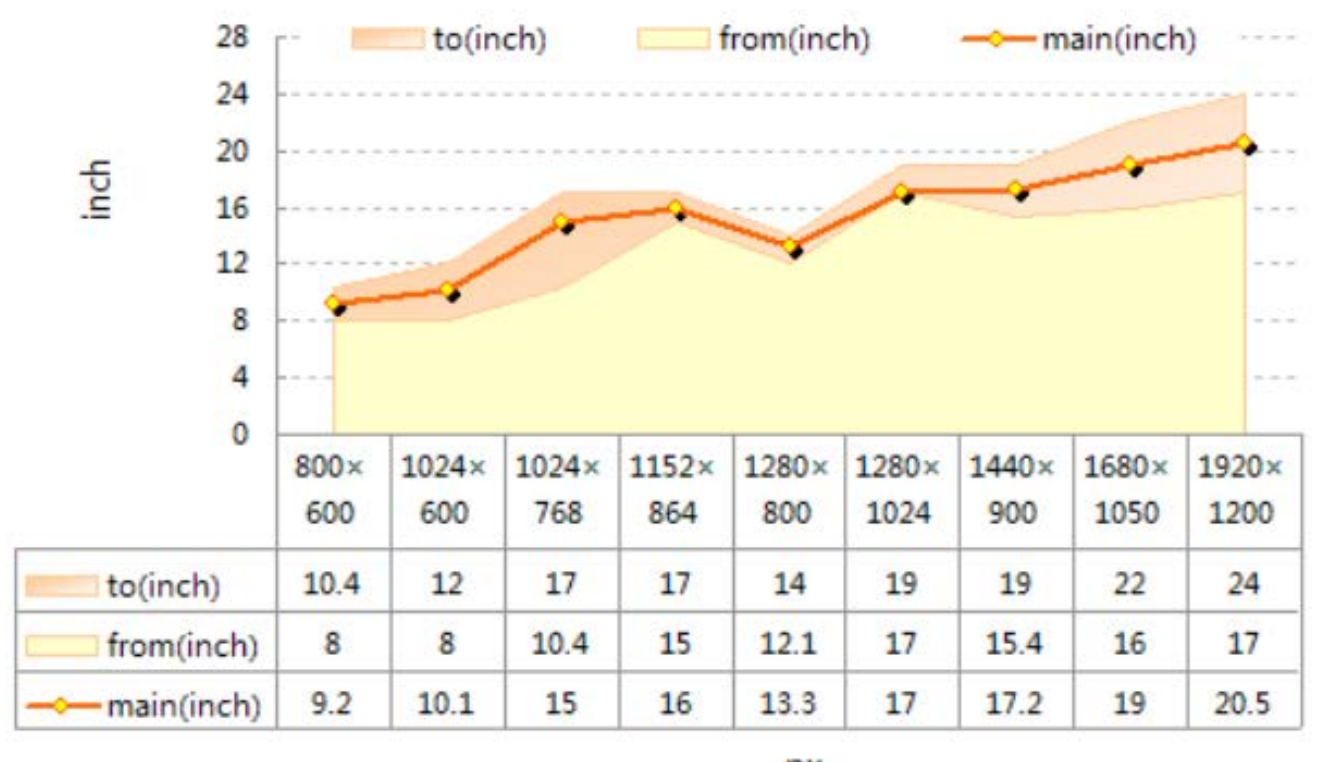

$\mathrm{px}$

Now the display aspect ratio not only 4:3 (standard screen),16:10(wide screen),but also more more kinds of irregular proportion. In order to compare displays with different aspect ratios, lateral resolution are used as the unified statistics. The width of the screen is calculated by the mainstream size and the aspect ratio , and then converted into a metric system, the trend of the screen size can 
be seen when corresponding them one by one.Coupled with the resolution of user distribution, the results shown in the figure below (In the picture, each point corresponds to a resolution, the horizontal axis is the horizontal screen pixels, the vertical axis is the physical width of the screen,the bubble size refers to the number of resolution users. The blue bubbles represent the standard screen, and the purple represents the wide screen.) The three biggest bubbles are $1024 * 768,1280 * 1024,1280 * 800$ [9]. Then calculate the physical width of the main screen size for each resolution, as shown in table 4 .

Table 1:The relationship between the pixel width and the physical width of the main monitor and the user's map.

According to the above relevant methods and the main audience group of the youth theme website, it is determined that the font size of this webpage is $14 \mathrm{px}$.

\subsection{Background login interface}

The initial interface of the website background is shown in figure 1.

\section{Summary}

Page design should be beautiful, generous, concise and maintain a consistent style. In order to achieve the best visual performance, the rationality of the overall layout should be emphasized, so that the visitors have a smooth visual experience. The main function of web pages is to provide a form for each user to understand the information provided by the site. To study web design, you need to stand in the user's perspective. Users are the key to a website's success or failure. If it takes a lot of time for users to access to the site, it's very likely that he or she may immediately turn off the site. Moreover, if the website operation is not very convenient for the user, they also will leave at once. All that mentioned above is a failure design because users will only be disappointed to leave. So web page capacity, also the number of pages (including images) is preferably no more than $50 \mathrm{~K}$.

\section{References}

[1] Internet Society of China, China Internet Network In $\square$ formation Center. 2012 China Internet Development Events(Sort by Time)[J]. China Internet, 2013(8): 68—69.

[2]LYU Xi. Practice and Research of Information Archi $\square$ tecture and Visualization Design Course[J]. Zhuangshi, 2012(11): 125-126.

[3]ZHOU Rui. The Interaction Design of Dynamic Effi $\square$ ciency in Starting State Interface[J]. Packaging Engi neering, 2015, 36(8): 83-86.

[4]YU Kang-kang, ZHANG Yu-hong. Inquiry into the Load $\square$ ing Design in APP Basic on the Augmented Reality Tech nique[J]. Art and Literature for the Masses, 2015(2): 97.

[5]ZHAO Meng-qi. Introduction to "Flat Design" in the Application of Interaction Design[J]. Design, 2014(3): 177-178.

[6]QIN Sui-ming, HE Meng-nan. Application of Flat Style in Web Interface Design[J]. ackaging Engineering, 2015, 36(12): 41-44.

[7]MA Tao. Material Texture and Texture Analysis in the Product Design[J]. Furniture \& Interior Design, 2016(3): 20-21.

[8]LI Jiang-yong. Research of Color Scheme for Construc $\square$ tion Machine Based on Brand Identity[J]. Journal of Graphics, 2014, 35(3): 423-428. 